\title{
Full ceramic restorations in posterior teeth
}

\author{
Marginal adaptation of different types of all-ceramic partial coverage restorations after exposure to an \\ artificial mouth
}

\section{F. J. Stappert, N. Denner, T. Gerds and J. R. Strub Br Dent J 2005; 199: 779-783}

\section{Objectives}

To determine the influence of the preparation design and the dimensions of all-ceramic partial coverage restorations (PCR) on the marginal accuracy before and after masticatory simulation.

Methods

In this in vitro study 80 extracted human maxillary molars were restored with MOD inlay restorations and four different modified PCR restorations using a new press ceramic IPS e.max ${ }^{\circledR}$ Press (IPS e.max ${ }^{\circledR}$ Press VP 1989). The teeth were divided into five groups of 16 specimens each and prepared as follows: Group A received an MOD inlay preparation and Group B, C, D and E received modified PCR. The restorations were adhesively luted and exposed to a mastication simulator. The discrepancies of the marginal fit were examined on epoxy replicas before and after luting as well as after masticatory simulation at 200x magnification.

Results

The mean (geometrical) [95\% confidence limits] marginal gap decreased from Group A to E before cementation (A-83[77-90] $\mu \mathrm{m}, \mathrm{B}-68[65-70] \mu \mathrm{m}$, C-59[55-64] $\mu \mathrm{m}, \mathrm{D}-56[52-61] \mu \mathrm{m}, \mathrm{E}-50[45-55] \mu \mathrm{m})$. Group A had significantly higher marginal gap values than group $B(p=0.017)$ and the other groups $(p<0.0001)$. After cementation the marginal accuracy was recorded as following: A-103[93-114] $\mu \mathrm{m}, \mathrm{B}-101$ [94-108] $\mu \mathrm{m}, \mathrm{C}-$ 93[89-98] $\mu \mathrm{m}, \mathrm{D}-102[98-105] \mu \mathrm{m}$ and E-99[96-102] $\mu \mathrm{m}$. Cementation increased the marginal gap in groups B-E significantly $(p<0.00001)$, not significantly in group $A(p=0.059)$. Artificial aging $(A-116[106-127] \mu m$, B-114[109-120] $\mu \mathrm{m}, \mathrm{C}-106[103-110] \mu \mathrm{m}, \mathrm{D}-109[100-118] \mu \mathrm{m}$ and E$109[105-112] \mu \mathrm{m})$ led to further significant decrease of marginal accuracy in Group B $(p=0.029)$ and C ( $p=0.026)$ only. After cementation and masticatory simulation of the ceramic restorations, the marginal gap values of Groups A, B, C, D and E did not significantly differ from each other $(p=1.00)$.

Conclusions

The result of this in-vitro study showed that IPS e.max ${ }^{\circledR}$ Press can be used to fabricate all-ceramic inlays and PCR which meet the requirements in terms of a clinically acceptable marginal gap, irrespective of the preparation design used. However, the preparation design and dimensions of the restorations appeared to affect the initial marginal fit and flowing off of luting material during the cementation process. The factors responsible for these findings require further substantiation.

\section{IN BRIEF}

- In general a significant decrease in marginal accuracy should be taken into consideration for partial coverage restorations after adhesive cementation.

- Adhesive luted partial coverage restorations appear to have a moderate increase in marginal gap width under masticatory aging

- The introduced new preparation designs fulfil the technical demands to fabricate accurate fitting all ceramic partial coverage restorations.

\section{COMMENT}

Modern dentistry wants to fulfil the aesthetic demands of the patients. Additionally preservation of the sound tooth substance is a very high concern. Tooth-coloured materials are used to imitate nature and according to adhesive technique retentive preparation is not needed anymore. The design of cavities, restored with adhesive restorations, is primarily based on size and localisation of the caries process. Only the needs of the material used, eg adequate thickness and rounded preparation design, have to be followed. Therefore, preparation designs vary heavily and in many cases it is not known how these different designs influence the clinical outcome of the restorations. The present study investigated five preparation designs for full ceramic restorations in posterior teeth.

Ceramic used for the restoration of a posterior tooth has to meet various criteria. For inlay and partial coverage crowns a high translucency is desirable. In addition, good physical properties are a prerequisite for clinical success. Empress 2 ceramic (Ivoclar Vivadent AG, Schaan, Liechtenstein) is a lithium disilicate ceramic with very good physical properties, improved compared with Empress 1, however with a relatively high opacity. Therefore it is of great interest to have data about the new pressed ceramic (IPS e.max ${ }^{0}$ Press, VP 1989, Vivadent AG, Schaan, Liechtenstein) with similar capacity and improved translucency compared with Empress 2. Colour of the restored tooth is a result of tooth colour, restoration colour and their interaction. Not only similar colour but also similar translucency of the tooth and the ceramic are very important to get a natural appearance of a restoration.

First step before the implementation of a new material should always be an in vitro test under conditions simulating the clinical situation. Using an artificial mouth for aging restorations is a good experimental design to imitate the oral cavity. This machine is able to simulate chewing at the same time with thermal cycling. The simulation of five years of clinical service with 1.2 million load cycles gives a good base for the clinical test.

The results of the present study showed that the larger and less retentive the preparation design was the better was the marginal fit before insertion, according to less retentive surfaces and according to this less jamming of the restoration. This result support the view that preparation angle for adhesively fixed restorations should not be too small. As masticatory simulation did not result in any destructive effects and marginal gap values did not differ significantly between the different preparation designs, practising very different designs should result in a successful restoration if the material parameters are harmonised with the requirements of the material used. Clinical studies will show if the improvement of the physical properties of the new ceramic will be able to reduce chipping fractures of the restoration which are observed relatively frequent in all ceramic restorations.

P. Hahn, Associate Professor at the Albert-Ludwigs-University Freiburg, University Clinic, Department of Operative Dentistry and Periodontology

doi: $10.1038 /$ sj.bdj.4813037 